\title{
REPETITIVE DISCHARGES FROM HUMAN MOTOR NERVES AFTER ISCHAEMIA AND THEIR ABSENCE AFTER COOLING
}

\author{
BY
}

\author{
WILLIAM COBB and JOHN MARSHALL
}

\author{
From the Department of Applied Electro-Physiology and the Neurological Research Unit of the \\ Medical Research Council, the National Hospital, Queen Square, London
}

The phenomena which occur in nerves in the post-ischaemic state may be those observed subjectively as paraesthesiae, or those of which objective evidence is provided by the electromyogram as repetitive muscle action-potentials. Both are believed to be due to iterative discharges occurring in the nerve, either spontaneously or in response to action currents evoked by normal or electrical stimuli. In both groups, however, despite many ingenious experiments, authors have disagreed on the site of origin of the repetitive discharges. The purpose of the present study was to seek further evidence on this point, and to investigate the possibility that similar discharges occur during cooling and the subsequent return of the nerve to normal temperature.

Post-ischaemic paraesthesiae were discussed by Reid (1929, 1931), who thought that they arose in the sensory end-organs. Lewis, Pickering, and Rothschild (1931), Bazett and McGlone (1932), and Zotterman (1933) also observed them but thought that they arose in the nerve fibres themselves. A technique commonly used to study this problem is to apply two inflated cuffs to a limb, one above the other, deflating the proximal cuff first, so that the circulation returns only to the segment of nerve beneath it. With this method Merrington and Nathan (1949) reported typical paraesthesiae, and Kugelberg (1946) recorded repetitive muscle actionpotentials. They concluded that the site of origin of these phenomena was in any segment of nerve recovering from ischaemia. Weddell and Sinclair (1947) also found paraesthesiae under these conditions, but considered them to be less intense than when the circulation was restored to the whole limb. They concluded that it is the return of the blood supply to nerve-endings which produces postischaemic paraesthesiae. Magladery, McDougal, and Stoll (1950), also using double cuffs, recorded repetitive muscle action-potentials while the distal cuff was still in place. They argued that a cuff on the forearm would allow blood to leak past, and hence the repetitive muscle action-potentials could not be assumed to be due to impulses arising in the segment of nerve under the proximal cuff. This criticism was answered by Kugelberg and Cobb (1951) who showed that release of pressure, just sufficient to permit a palpable pulse at the wrist for six minutes, was insufficient to cause repetitive responses, though they occurred readily when the circulation was completely released.

Another experimental technique, which might have been expected to give a definite answer to the problem, was to occlude the circulation in an amputation stump. Following such an occlusion Weddell and Sinclair found no paraesthesiae in the phantom limb, whereas two out of Merrington and Nathan's seven subjects reported paraesthesiae. The latter authors suggest that failure to obtain paraesthesiae in some cases is probably due to the practice of dividing nerves as high as possible during amputation. Thus, unless a cuff can be placed high on the stump, the nerve trunk may not be rendered ischaemic.

Localized compression of a segment of nerve without occlusion of the peripheral circulation has also been employed as a method of study. Again Merrington and Nathan found typical paraesthesiae, while Weddell and Sinclair reported none. The former authors made it clear that a greater length of nerve must be made ischaemic to cause paraesthesiae than that required to produce sensory loss. Recordings of muscle action-potentials after ischaemia of a short segment of nerve have not been reported.

The importance of this subject is more than merely academic for ischaemia is being used increasingly as a means of studying clinical problems (Bharucha, 1952 ; Marshall, 1952; Gilliatt and Wilson, 1953), so that accurate knowledge of the site of origin of these phenomena is required.

If a segment of nerve can be rendered ischaemic 
without impairment of the circulation to the nerve endings, then positive phenomena occurring after ischaemia must arise in that segment of nerve. The original nerve clamp of Lewis and others (1931) was somewhat unpredictable in its action, but even so, in satisfactory experiments, no question of impediment to the peripheral circulation arose. The modified clamp (Marshall, 1953) is much more reliable, and any obstruction to the circulation can be confidently rejected. Hence, in the study reported here a nerve clamp was used to produce local ischaemia in nerve fibres. The effects during and after cooling the nerve were separately observed.

\section{Methods}

The observations were made on six healthy adult subjects.

The electromyographic recordings were made through a coaxial needle electrode inserted into the first dorsal interosseous muscle. The action-potentials were amplified by a differential amplifier with an adequate range of frequency response, and displayed on one beam of a double-beam cathode ray oscillograph. The other beam was fed with a time scale giving intervals of 1 and 10 m. sec., derived from a crystal oscillator.

Cathodal stimulation of the ulnar nerve was applied as high in the upper arm as possible or at the wrist. The voltage pulse of the stimulus had an exponential decay with a time constant of $20 \mu \mathrm{sec}$, , and the stimulus was made supra-maximal, as judged from the amplitude of
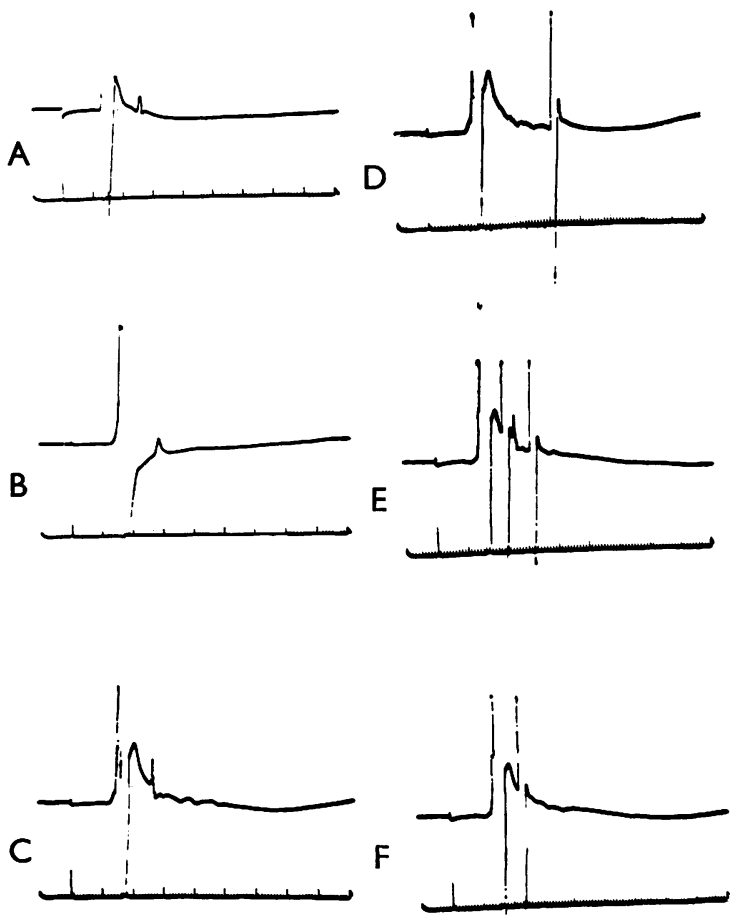

the summated muscle action-potential at the beginning of the experiment. The stimulus was triggered once per second by the time scale, at a point indicated on it.

Ischaemia of the limb was produced by a sphygmomanometer cuff, $12.5 \mathrm{~cm}$. wide, inflated to $200 \mathrm{~mm}$. $\mathrm{Hg}$, placed around the arm immediately above the elbow, and below the point of stimulation of the nerve in the upper arm.

Ischaemia of a segment of the ulnar nerve immediately above the elbow was produced by a modified nerve clamp (Marshall, 1953) $12.5 \mathrm{~cm}$. long ; it was inflated to $100 \mathrm{~mm} . \mathrm{Hg}$, this pressure having been found to give rise to ischaemic and post-ischaemic changes without any impairment of the general circulation to the limb.

Cooling of a segment of the ulnar nerve at about the same level was obtained by placing the flexed elbow in a mixture of ice and water at 0 to $1^{\circ} \mathrm{C}$., the electrical stimulus being applied proximal to the cooled area.

\section{Results}

All the illustrations are from experiments on the same subject ; there are small variations in latency from subject to subject but those illustrated are typical and are quoted alone for simplicity.

Observations before Ischaemia or Cooling.Following a stimulus in the upper arm there is a latent period of about $12 \mathrm{~m}$. sec. before the occurrence of the primary response in the first dorsal interosseous muscle (Fig. 1a). At about $25 \mathrm{~m}$. sec. a further, much smaller, response occurs, which is the F wave of Magladery and others (1950); this is extremely variable in its occurrence and may be barely visible or relatively large and sharp ; in any subject, for a given electrode placement, it has several forms, each of which may be recognized by the wave shape. The limited number of constant forms of response suggests that each is the product of one motor unit, the form and size depending on the relation of this to the needle electrode.

Fig. 1a.-A single cathodal stimulus to the ulnar nerve high in the upper arm gives rise to a primary response from first dorsal interosseous muscle with a latency of $12(\cdot 2) \mathrm{m}$. sec. followed by an $F$ wave with a latency of $24(\cdot 5) \mathrm{m}$. sec.

FIG. 1b.-After $17 \mathrm{~min}$. ischaemia, produced by an inflated cuff on the arm, the primary latency has increased to $13(.5) \mathrm{m}$. sec., and the $\mathrm{F}$ latency to $27(.5) \mathrm{m}$. sec.

FIG. 1c.-The cuff was released immediately following B, and $2 \mathrm{~min}$. later a small repetitive response begins and is seen in all subsequent records, up to seven minutes.

Fig. 1d.-After a further $2 \mathrm{~min}$. a high-voltage spike occurs following each stimulus, at first with a latency of $40 \mathrm{~m}$. sec.

Fig. 1e.-The latency of this spike decreases and it is shortly followed by another identical spike. In this example the $F$ wave lies between the two spikes.

Fig. If.-The second spike no longer occurs and the latency of the first has fallen to $22 \mathrm{~m}$. sec.; it also ceases shortly afterwards.

Time-scale for all figures 1 and $10 \mathrm{~m}$. sec. Stimulus at $10 \mathrm{~m}$. sec. (For calibration see Fig. 3). 

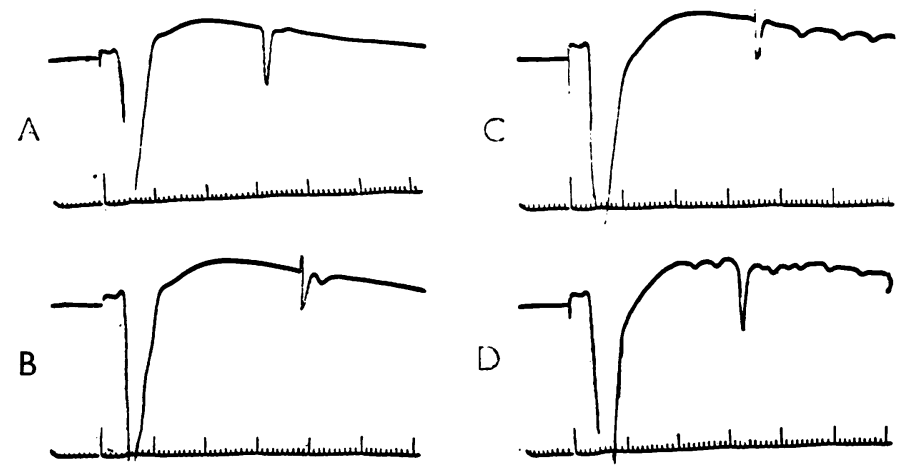

Fig. 2a.-As for 1a, but the stimulus applied to the ulnar nerve at the wrist. The primary latency is $2(.8) \mathrm{m}$. sec. and the F latency $32 \mathrm{~m}$. sec.

FIG. 2b.-After inflation of the cuff on the arm for $14 \mathrm{~min}$. there is no change in the primary latency, while that of the $F$ wave has increased to 38 m. sec.

FIG. 2c.-About $2 \mathrm{~min}$. after release of the cuff a repetitive response occurs, at first following the F wave.

FIG. 2d.-Several minutes later, when the $F$ latency has returned to its original value, repetitive responses occur both before and after the $F$ wave.

Note the two distinct forms of $F$ wave.
Following stimulation at the wrist the primary response has a latency of about $3 \mathrm{~m}$. sec. while the $\mathrm{F}$ wave is delayed to about $32 \mathrm{~m}$. sec. (Fig. 2a).

Observations after Ischaemia Produced by a Cuff.The events which occur during and after occlusion of the circulation to the whole limb distal to a cuff on the arm are illustrated in Figs. 1 and 2. Fig. 1a shows the response to stimulation of the ulnar nerve above the cuff before ischaemia. After ischaemia for $17 \mathrm{~min}$. the latencies of the primary response and $F$ wave have become $13(\cdot 5)$ and 27 $(.5) \mathrm{m}$. sec. respectively (Fig. 1b) ; these are smaller increases than were obtained in some of the subjects for comparable durations of ischaemia, up to 4.5 $\mathrm{m}$. sec. for the primary response being noted.

Two minutes after release of the cuff at $17 \mathrm{~min}$. small repetitive responses begin, shown in Fig. 1c following the $F$ wave, whose latency has fallen to $26 \mathrm{~m}$. sec. After a further two minutes a high voltage triphasic action-potential appears, and recurs after every stimulus ; at first its latency is $40 \mathrm{~m}$. sec. (Fig. 1d), but this steadily drops and it is shortly followed by a second identical spike seen in Fig. 1e with the $F$ wave between them. After being evoked by a number of successive stimuli the second spike drops out (Fig. 1f) and later the first also. During the whole of this time the initial small response has continued, but finally ceases about seven minutes after release of the cuff.

Fig. 2 illustrates the results of stimulation at the

\section{Fig. 3a.-As for Fig. 1a.}

FIG. 3b.-After inflation of a nerve-clamp on the ulnar nerve above the elbow for $19 \mathrm{~min}$. both latencies are slightly increased.

FIG. 3c.-Following a release of the clamp at $20 \mathrm{~min}$. a repetitive response occurs from about the first to seventh minute. Seven successive sweeps at 1 sec. intervals show the great variability in timing of the response.

FIG. 3d.-Calibration $=1 \mathrm{~m} . \mathrm{V}$. wrist ; the subject is the same as in Fig. 1 but the occasion different.

In Fig. 2a, before ischaemia, the latency of the primary response is $2(\cdot 8) \mathrm{m}$. sec. and that of the F wave $32 \mathrm{~m}$. sec. After inflation of the cuff on the arm for $14 \mathrm{~min}$. there is no detectable change in the primary latency but that of the $\mathrm{F}$ wave has increased to $38 \mathrm{~m}$. sec. (Fig. 2b). During this experiment two forms of $F$ wave consistently appeared as shown in $a$ and $b$.

Following release of the cuff repetitive responses appear from about the middle of the second minute to the eighth ; at first (Fig. 2c) they only follow the $F$ wave, but later occur both before and after it (Fig. 2d) at a time when the $F$ latency has returned to its initial value.
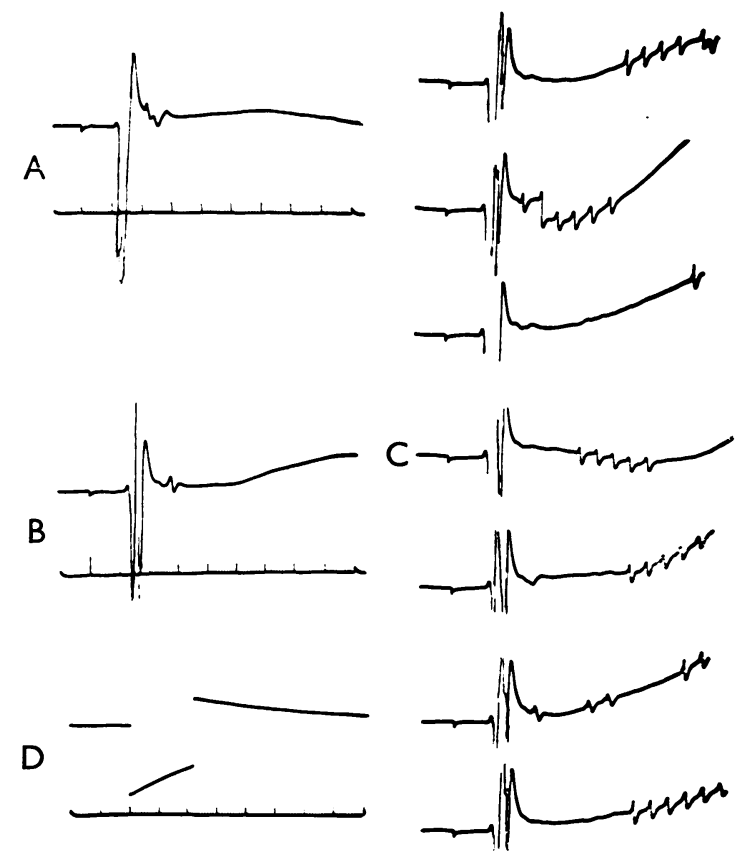


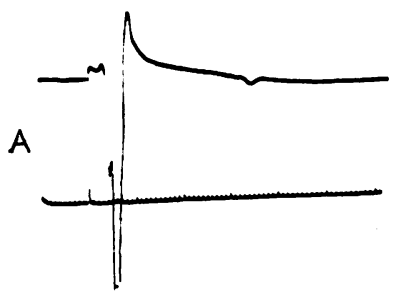

Fig. 4a.-As for Fig. 2a.

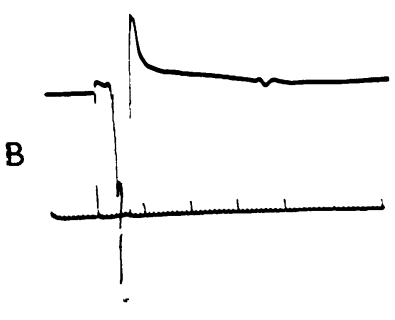

Fig. 4b.-The nerve-clamp has been inflated for 18 min. and the $F$ latency is slightly increased.

Fig. 4c.-Several minutes after release of the clamp repetitive discharges occur after the $F$ wave. They were never seen before it.

Observations after Ischaemia Produced by a Nerve-clamp. - When the clamp was applied to the ulnar nerve at a pressure of $100 \mathrm{~mm}$. $\mathrm{Hg}$ no impediment to the arterial supply to or venous return from the limb could be detected. Stimulation was carried out exactly as in the previous experiments and typical results are illustrated in Figs. 3 and 4. In Fig. 3a, before inflation of the clamp, the primary response has a latency of $11 \mathrm{~m}$. sec. and the $F$ wave, which is not well differentiated from it, of $23 \mathrm{~m}$. sec.

After compression of the nerve for $19 \mathrm{~min}$. the primary latency has risen to $12 \mathrm{~m}$. sec. (Fig. 3b) and the $F$ latency to $25.5 \mathrm{~m}$. sec. In all the clamp experiments the changes in latency were less than for the cuff experiments on the same subjects.

The pressure was released at $20 \mathrm{~min}$. and repetitive responses began about one minute later. Fig. 3c shows the results of seven successive stimuli, in which it can be seen that the time distribution of the responses varies considerably and in no predictable manner, although the constancy of wave form suggests that it is the same unit firing in every case.

On the occasion when this same subject was used for the clamp experiment with stimulation at the

wrist, both the $F$ wave and repetitive responses were small, though perfectly clear and consistent. Fig. 4a shows a primary latency of $2(\cdot 8) \mathrm{m}$. sec. and an $F$ latency of $33 \mathrm{~m}$. sec. before inflating the clamp. After compression for $18 \mathrm{~min}$. the primary latency is unchanged (Fig. 4b) and the F latency has risen to $35 \mathrm{~m}$. sec. Release of the clamp at this time was followed for a longer interval without repetitive responses than in the cuff experiments, but they then recurred for several minutes, up to eight minutes after release of the clamp. At no time were they seen to occur before the $F$ wave (Fig. 4c), or its expected time when it was not actually measurable.

Observations during and after Cooling of a Nerve Segment.-By placing the flexed elbow in ice-water a segment of the ulnar nerve in the region of the elbow, by reason of its superficial position, was subjected to cold. This segment was approximately the same in length as that compressed by the cuff and clamp but possibly a little more distal. As the sites of stimulation and recording were the same as during ischaemia, the experimental conditions were nearly identical.

Fig. 5a shows the response to stimulation in the arm before the elbow was inserted in the ice; the

Fig. 5a.-As for Fig. 1a. Primary latency $12.5 \mathrm{~m}$. sec.; F latency $26.5 \mathrm{~m}$. sec.

FIG. 5b.-The flexed elbow has been placed in icewater to cover about 12 $\mathrm{cm}$. from the elbow proximally. Stimulation of the ulnar nerve in the upper arm during the 30 -min. period of immersion causes no unusual responses, while the latencies rise to 18.5 and 35 m. sec.

Fig. 5c.-Stimulation at frequent intervals during the $20 \mathrm{~min}$. following removal of the elbow from the ice-water caused no repetitive discharge.
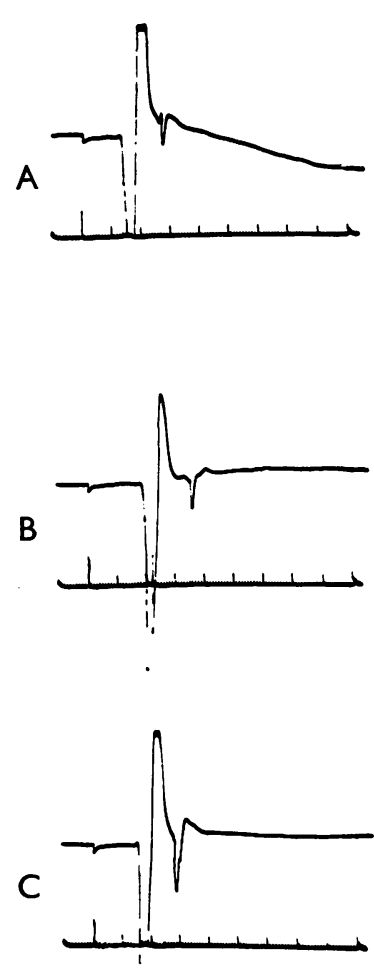
primary latency is $12.5 \mathrm{~m}$. sec. and the $\mathrm{F}$ latency $26.5 \mathrm{~m}$. sec. Stimulation is continued at $1 \mathrm{sec}$. intervals while the elbow remains in the ice for 30 min. (the period varying from 10 to $30 \mathrm{~min}$. in the different trials). During cooling no major change is noted in the muscle action-potential except an increase in primary latency to $18.5 \mathrm{~m}$. sec. and in $\mathrm{F}$ latency to $35 \mathrm{~m}$. sec. (Fig. $5 \mathrm{~b}$ ).

The elbow is then removed from the ice and stimulation continued at intervals to $20 \mathrm{~min}$. The latencies return to the initial values more slowly than after ischaemia, being still increased at $15 \mathrm{~min}$. (Fig. 5c). At no time in any subject was there any suggestion of a repetitive response. In the experiment illustrated immersion in ice-water was continued for longer than in any other and the changes in latency are more extreme than in them; the rate of recovery is correspondingly slower.

Stimulation at the wrist was not performed in view of the absence of any repetitive response.

\section{Discussion}

The cuff experiments were a repetition of the work of several authors and were done to provide a control with which to compare the nerve-clamp and cooling experiments ; they do, however, bring out several points of importance in the discussion of this subject.

The variations in form of the $F$ response have already been mentioned, with reasons for supposing that they must be due to the discharge of different units having varying relationships to the needle. As the $F$ wave is always smaller and briefer than the primary response it is evident that it can only represent a fraction of the total units within range of the needle; which unit actually fires is presumably determined by fluctuations in excitability within the available neuron pool.

The repetitive responses during post-ischaemia show the same, or an even wider, variation in form, but here again it is usually possible, with the more sharply defined examples, to recognize that at any time it is probably only one motor unit which is firing, or sometimes two or three ; this is particularly clearly seen in Fig. 1, which shows the largest response in the whole series in great contrast with the other response in the same figure, which presumably occurred in a more distant unit.

This figure also shows clearly the possibility of changes in latency of the repetitive response, which in this case at least became shorter throughout the period during which this response occurred though with a not quite constant progression. By contrast, the timing of the repetitive response in Fig. 3c shows a completely random scatter.
The importance of these considerations is that comparisons between the responses to different experimental techniques cannot be made on the basis of their clarity ; the cuff may be more evocative of a repetitive response than the clamp but the differences between Figs. 1 and 3 are not evidence for it. Similarly, care must be exercised in making deductions from the latency of the repetitive response, which is subject to wide variation, though on the whole tending to shorten during the period of recovery.

When the nerve-clamp was used the pulses in the forearm were full and free, blanching of the nail-bed on pressure was followed by an immediate return of the red blush, and the veins showed no distension. Furthermore, the ischaemic and post-ischaemic subjective changes were confined to the ulnar fingers ; one subject, deluded by the lack of such changes in the index finger, was surprised to find, after compression for only $17 \mathrm{~min}$., that he had complete motor nerve block to the first dorsal interosseous muscle. It can be assumed, therefore, that th.e properly positioned nerve-clamp renders ischaemic a segment of the ulnar nerve which is probably about the same length as that of the clamp and certainly does not extend to the nerve endings. Comparison of the changes in latencies of the primary response and $F$ wave in the cuff and clamp experiments is consistent with this view, but they could be due to different degrees of coincident cooling of the hand and forearm.

It is clear that in the clamp experiments the repetitive responses must have arisen in the length of nerve under the clamp, the only portion to have been ischaemic, and not at the myoneural junctions. Further evidence for the purely local nature of the ischaemia, and hence of the hyperirritability, is provided by the timing of the repetitive responsc. When the whole forearm has been ischaemic and a stimulus is applied to the ulnar nerve at the wrist repetitive responses might be expected to occur immediately following the primary response, and this was sometimes observed. On the other hand, when using a clamp at the elbow, a stimulus at the wrist would be applied to normal nerve and no immediate repetitive response could be expected, nor was it seen.

Although the three experimental techniques were as nearly similar as possible it was only after cooling that a repetitive response could not te seen at all. It seems unlikely that this absence was due to inadequate cooling, for the increase in latency of the primary response was about $50 \%$. A possible explanation is that the changes in the nerve responsible for repetitive discharges take place more 
slowly after cooling than after ischaemia. Thus, after cooling it required ten minutes or more for the latency of the response to return to its original value, whereas after ischaemia the average time was about five minutes. It may, therefore, be the relative rapidity of the changes in the nerve which causes spontaneous firing in the post-ischaemic state. In a somewhat similar experiment Pinelli and Buchthal (1953) kept the hand warm and recovery was then much more rapid, although the duration of cooling was one hour and its degree probably greater. Like us they did not notice any spontaneous or evoked repetitive response, in this case to the natural stimulus of voluntary muscular contraction.

A more probable reason is that ischaemia and cold have fundamentally different effects on nerve fibres. This would accord with the difference in the order of loss of sensory modalities (Sinclair and Hinshaw, 1951), and in the behaviour of the pain threshold (Marshall, 1953) which have been observed in ischaemia and in cooling of a nerve. The subjective changes after ischaemia and after cooling have also entirely different qualities.

It may be concluded, therefore, that the phenomena observed in nerves after ischaemia arise not in the nerve endings but in that segment of the nerve trunk which was rendered ischaemic. Interpretation of tests which use ischaemia produced by a cuff or by a clamp need to be made in the light of this fact. Cooling a similar segment of nerve does not produce these phenomena, possibly because the effects of cold and ischaemia on nerve fibres are fundamentally different.

\section{Summary}

Further evidence is presented on the site of origin of the repetitive discharge in (human) motor nerves following ischaemia, as revealed by the electrical discharge in muscle.
During the recovery period following ischaemia, produced by inflation of a pneumatic cuff round the arm, repetitive discharges are readily evoked in the first dorsal interosseous muscle by electrical stimulation of the ulnar nerve.

A similar result may be obtained by local pressure, exerted by a nerve-clamp on the same segment of the ulnar nerve, although, during compression, there is no evidence of interference with the general circulation of the forearm and hand.

It is concluded that the repetitive discharge arises in that segment of the nerve which has . been ischaemic.

After a similar length of nerve has been cooled by immersion of the elbow in ice-water for up to 30 minutes no repetitive discharge can be evoked.

This supports the subjective evidence that the effects of ischaemia and cooling on nerve are qualitatively and probably fundamentally different.

Our thanks are due to Prof. Eric Kugelberg and Dr. P. A. Merton for helpful criticism and to Mr. H. B. Morton for his technical assistance, also to Dr. E. A. Carmichael, Director of the Neurological Research Unit, for his encouragement.

\section{REFERENCES}

Bazett, H. C., and McGlone, B. (1932). Arch. Neurol. Psychiat., Chicago, 28, 71 .

Bharucha, E. P. (1952). Clin. Sci., 11, 233.

Gilliatt, R. W., and Wilson, T. G. (1953). Lancet, 2, 595.

Kugelberg, E. (1946), Brain, 69, 310. and Cobb, W. (1951). Journal of Neurology, Neurosurgery

Lewis, T., Pickering, G. W., and Rothschild, P. (1931). Heart, 16, 1.

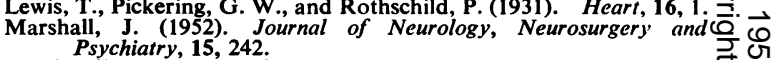

- (1953). Clin. Sci., 12, 247.

Magladery, J. W., McDougal, D. B., and Stoll, J. (1950). Johns.

Hopk. Hosp. Bull., 86, 313.
Merrington, W. R., and Nathan, P. W. (1949). Journal of Neurology, Neurosurgery and Psychiatry, 12, 1

Pinelli, P., and Buchthal, F. (19:3). Electroenceph. Clin. Neuro-

Reid, C. (1929). 5, 589. Quart. J. exp. Physiol., 19, 127.

(1931). Ibid., 21, 243.

Sinclair, D. C., and Hinshaw, J. R. (1951). Brain, 74, 318.

Weddeli, G., and Sinclair, D. C. (1947). Journal of Neurology, Neurosurgery and Psychiatry, 10, 26.

Zotterman, Y. (1933). Acta med. scand., 80, 185. 